OPEN ACCESS

Edited by:

Dirk M. Hermann,

Universität Duisburg-Essen, Germany

Reviewed by:

Ertugrul Kilic,

Istanbul Medipol University, Turkey

Carmelo Chisari,

Azienda Ospedaliero Universitaria

Pisana, Italy

*Correspondence:

Yan-Mei Yang

yym9876@sohu.com

†These authors have contributed equally to this work.

Received: 09 February 2018 Accepted: 18 July 2018

Published: 07 August 2018

Citation:

Hao X-Z, Yin L-K, Tian J-Q, Li C-C,

Feng $X-Y$, Yao $Z-W$, Jiang $M$ and Yang Y-M (2018) Inhibition of Notch1

Signaling at the Subacute Stage of Stroke Promotes Endogenous Neurogenesis and Motor Recovery After Stroke.

Front. Cell. Neurosci. 12:245 doi: 10.3389/fncel.2018.00245

\section{Inhibition of Notch1 Signaling at the Subacute Stage of Stroke Promotes Endogenous Neurogenesis and Motor Recovery After Stroke}

\author{
Xiao-Zhu Hao ${ }^{1 t}$, Le-Kang Yin ${ }^{2 t}$, Jia-Qi Tian', Chan-Chan Li', Xiao-Yuan Feng ${ }^{1}$, \\ Zhen-Wei Yao' ${ }^{1}$, Min Jiang ${ }^{3}$ and Yan-Mei Yang ${ }^{1 *}$ \\ 'Department of Radiology, Huashan Hospital, Fudan University, Shanghai, China, ${ }^{2}$ Department of Radiology, Shanghai \\ Chest Hospital, Shanghai Jiaotong University, Shanghai, China, ${ }^{3}$ Institutes of Brain Science and State Key Laboratory of \\ Medical Neurobiology, Fudan University, Shanghai, China
}

Background and Purpose: It is still not clear whether Notch1 signaling inhibition can promote functional outcomes after stroke, given that it plays time-dependent roles in the sequential process of endogenous neurogenesis. The purpose of this study was to identify the appropriate time frame for Notch1 signaling inhibition according to the temporal evolution of Notch1 signaling activation and the responses of neural stem cells (NSCs), in order to target it for therapeutic intervention and stimulate neurorestorative strategies after stroke.

Methods: Sprague-Dawley (SD) rats were subjected to 90-min of middle cerebral artery occlusion (MCAO). Rats were sacrificed before, and at day 1 , day 2 , day 3 , day 4 , and day 7 after ischemia for immunohistochemical analysis of the Notch intracellular domain (NICD), Nestin and doublecortin (Dcx). Next, MCAO rats were treated with the $\gamma$-secretase inhibitor $N$-[N-(3,5-di uorophenacetyl)-1-alanyl]-S-phenylglycine t-butylester (DAPT) or with saline at day 4 after ischemia, and subsequently evaluated with behavioral test analysis and magnetic resonance imaging (MRI). The rat brains were then harvested for immunohistochemical analysis of Dcx, NeuN and myelin basic protein (MBP) at 2, 3, 4 , and 8 weeks.

Results: Notch1 signaling was maximally activated at day 3 after ischemia in parallel with the temporal evolution of NSCs. Inhibiting Notch1 signaling at day 4 after reperfusion with DAPT further promoted recovery of MRI parameters of the corticospinal tract (CST) and the functional outcomes, concomitantly with an increase in neuroblasts, their migration to the ischemic boundary, and potential differentiation to mature neurons, as well as the amelioration of axonal bundle integrity.

Conclusion: Inhibition of Notch1 signaling at the subacute stage of stroke could maximally promote endogenous neurogenesis and axonal reorganization.

Keywords: Notch1, neurogenesis, MCAO, DAPT, striatum, internal capsule, magnetic resonance imaging, stroke 


\section{INTRODUCTION}

Stroke is one of the leading causes of death and serious long-term disability (Stinear et al., 2007; Smajlović, 2015). Importantly, a large number of stroke patients are permanently disabled in that only a minority of patients can benefit from thrombolysis given its limited therapeutic time window. Novel neurorestorative therapies with a wider therapeutic window that can promote brain repair, are thus, urgently needed to enhance functional neurological recovery following stroke (Barone, 2010; Zhan et al., 2011). Previous studies have demonstrated that cerebral ischemia induces proliferation of NSCs in the SVZ, which migrate into the damaged brain regions, differentiate into mature neurons and ultimately integrate into local as well as remote neural circuits (Arvidsson et al., 2002; Zhang et al., 2008; Wang L. et al., 2009; Sun et al., 2013), suggesting that endogenous neurogenesis could be a target for rehabilitative therapy in stroke patients.

In recent years, Notch1 signaling, which is critical for endogenous neurogenesis, has been regarded as a potential therapeutic target for promoting functional recovery after stroke (Wei et al., 2011). Notch1 is expressed in NSCs and neuroblasts and its activity is fundamental for neural development as well as neural specification by controlling maintenance, proliferation and differentiation of NSCs in young and aged brain in normal or pathological conditions (Zhang et al., 2008; Wang X. et al., 2009; Sun et al., 2013). Interestingly, while some studies have found that Notch1 signaling activation could promote neurogenesis (Oya et al., 2009; Wang X. et al., 2009), others support the idea that Notch1 signaling negatively regulates neurogenesis (Li et al., 2012). Notably, preventing Notch1 cleavage into the Notch intracellular domain (NICD) with the $\gamma$-secretase inhibitor N-[N-(3,5-diuorophenacetyl)-1-alanyl]S-phenylglycine t-butylester (DAPT), subsequently improves functional outcomes following stroke (Li et al., 2012). Except for differences in animal strain and stroke models, the most plausible explanation for the conflicting results cited above is the spatio-temporal regulation of Notch activity (Zhao et al., 2012); in other words, Notch-1 signaling playing space and time-dependent roles in the sequential process of neurogenesis (Chambers et al., 2001). Moreover, several studies have found that Notch-1 signaling was activated in the acute stage of stroke to promote NSCs proliferation and was attenuated in the subacute stage to promote neuronal differentiation (Oya et al., 2009; Wang L. et al., 2009). Based on this standpoint, the detection of the temporal evolution of Notch1 signaling activation following

Abbreviations: AD, axonal diffusion; ANOVA, One-way analysis of variance; aSVZ, anterior subventricular zone; CCA, common carotid artery; CST, corticospinal tract; DAPT, $N$-[N- (3,5-difluorophenacetyl)-1-alanyl]-Sphenylglycine t-butylester; Dcx, doublecortin; DTI, diffusion tension imaging; ECA, external cerebral artery; FA, fractional anisotropy; FOV, Field of View; IC, internal capsule; ICA, internal cerebral artery; IF, immunofluorescence; LSD, least-significant difference; $\mathrm{LV}$, lateral ventricle; MBP, myelin basic protein; MCA, middle cerebral artery; MCAO, middle cerebral artery occlusion; MRI, magnetic resonance imaging; NA, number of average; NICD, Notch intercellular domain; NSC, neural stem cell; PBS, phosphate buffer saline; PFA, paraformaldehyde; RD, radial diffusion; ROI, region of interest; SD, standard deviation; SI, signal intensity; ST, slice thickness; STR, striatum; SVZ, subventricular zone; TE, echo time; TR, repetition time. cerebral ischemia and attempts to timely control its activation are required to augment the neural progenitor pool and promote neural differentiation to attain morphological and functional maturity in the adult brain.

Furthermore, it appears unreasonable and insufficient to define the beneficial or detrimental effects of therapeutic interventions of the Notch1 pathway based on in vitro pathological examinations. Thus, it is important to develop non-invasive methods to monitor modifications of brain tissue and predict long-term motor outcomes, which is essential to promote clinical applications of emerging neurorestorative therapies. Cross-sectional studies have demonstrated that diffusion tensor imaging (DTI) could provide unparalleled insights into the microstructural properties of central nervous system (CNS) tissue (Nucifora et al., 2007; Budde and Frank, 2012). For instance, diffusion parameter changes in the CST have been established as surrogate makers of motor deficit after stroke (Thomalla et al., 2005; Stinear et al., 2007; Lindenberg et al., 2012; Feng et al., 2015).

In this study, firstly, we aimed to detect the temporal evolution of Notch1 signaling activation and NSCs responses after stroke. Based on our results, we then attempted to find the appropriate therapeutic time frame for DAPT treatment. More importantly, for the first time, we measured the comprehensive microstructure changes in the CNS with a set of MR parameters, combined with the post mortem immunohistochemical analysis of neurogenesis and remyelination of the CST, and ultimately demonstrated the neurorestorative effects of DAPT treatment at the subacute stage after stroke.

\section{MATERIALS AND METHODS}

\section{Animals}

Adult male Sprague-Dawley rats (260-270 g) were obtained from the Shanghai Experimental Animal Center of Fudan University. All procedures performed in this study were approved by the Fudan University of Institutional Animal Care and Use Committee, and every effort was made to minimize suffering and to reduce the number of animals used (20171740A704).

\section{Experimental Groups}

For semiquantitative evaluation of Notch1 signaling activation and neural proliferative response at the early stage of stroke, rats were subjected to MCAO. Thirty-eight rats underwent the surgery and eight rats were excluded from our analysis because of death or poor lesions which is shown in the T2-weighted MR images $24 \mathrm{~h}$ after surgery (data not shown). In our study, five rats were sacrificed respectively before, and at day 1 , day 2 , day 3 , day 4 , and day 7 after the induced stroke, and subjected to immunohistochemical analysis of Nestin, Dcx, and the NICD.

To evaluate proliferative responses following treatment with the $\gamma$-secretase inhibitor, DAPT, at the subacute stage of stroke, rats were subjected to MCAO, treated by intracerebroventricular (i.c.v) administration (day 4) of DAPT or saline. Forty rats underwent surgery, of which three rats were not selected for further experiments because of poor lesions and five rats died 
during the course of our study. Therefore, eight rats of each subgroup were successively evaluated with behavioral tests and MRI, followed by tissue harvesting for immunohistochemical analysis of Dcx, NeuN, and MBP at 2, 3, 4, and 8 weeks respectively. Figure 1 shows the time schedule for experimental procedures in all groups (Figure 1).

\section{Stroke Model}

Rats were anesthetized with an intraperitoneal (i.p.) injection of $10 \%$ chloral hydrate under spontaneous inspiration with continuous body temperature monitoring $37^{\circ} \mathrm{C} \pm 0.5^{\circ} \mathrm{C}$, during the surgical procedures. For all rats, the left middle cerebral artery (MCA) was occluded for $90 \mathrm{~min}$. In details, rats were immobilized by a tooth holder and with binding of all limbs, followed by the insertion of a 4.0 silicon-coated polypropylene suture into the left internal carotid artery (ICA) through the external carotid artery (ECA) and CCA to block blood flow to the MCA. After $90 \mathrm{~min}$, the filament was withdrawn from the ICA to allow reperfusion. The follow-up T2 MRI was acquired under the anesthetized circumstances to check the occlusion of the left hemisphere $24 \mathrm{~h}$ post-stroke. The above procedure was identical to that of our previous study (Hao et al., 2016).

\section{Administration of $\boldsymbol{\gamma}$-secretase Inhibitor}

DAPT powder (Sigma-Aldrich, St. Louis, MO, United States), was dissolved in DMSO to prepare concentrations of $8.3 \mathrm{mg} / \mathrm{ml}$. DAPT solution $(0.03 \mathrm{mg} / \mathrm{kg})$ was stereotactically injected into the lateral cerebral ventricle (LV) for the DAPT treated group rats 4 days after the induced stroke. Rats were anesthetized and placed in a stereotactic holder and immobilized by earplugs and tooth holder. A burr hole was drilled in the skull, $4.0 \mathrm{~mm}$ deep into the pial surface, $-2.0 \mathrm{~mm}$ anteroposterior relative to the bregma, and $1.0 \mathrm{~mm}$ lateral to the midline, according to Paxinos and Watson (1998). With a $2.0 \mu$ l Hamilton syringe, DAPT administration was finished within $3 \mathrm{~min}$ and the needle was left in place for $4 \mathrm{~min}$ to prevent leakage along the injection track. Animals in the control group received the same volume of saline at the same time point.

\section{Magnetic Resonance Imaging}

Multimodal MRI of subject animals, including relaxation time imaging and diffusion imaging, was temporally conducted in each experimental group. Prior to MRI, animals were anesthetized by the same procedure as described for the MCAO model (see above). The body temperature was continuously monitored at $37^{\circ} \mathrm{C} \pm 0.5^{\circ} \mathrm{C}$, at the same time, blood oxygen saturation and heart rate were also monitored during MRI procedures. The MRI measurements were performed in a 3.0-T horizontal magnet (Discovery MR750, GE Medical Systems, Milwaukee, WI, United States) with a 60-mm-diameter gradient coil (Magtron Inc., Jiangyin, China).

T2-weighted MR images were acquired by a fast spin-echo sequence with the following parameters: $\mathrm{TR} / \mathrm{TE}=4,000 \mathrm{~ms} / 96 \mathrm{~ms}$, scan time $=3 \mathrm{~min}$, $\mathrm{FOV}=6 \mathrm{~cm} \times 6 \mathrm{~cm}$, matrix $=256 \times 256, \mathrm{ST}=1.8 \mathrm{~mm}$, spatial resolution $=0.24 \mathrm{~mm} \times 0.24 \mathrm{~mm} \times 1.8 \mathrm{~mm}$, inter-slice distance $=2 \mathrm{~mm}$, number of slices $=15$, and $\mathrm{NA}=2$.
Three-dimensional T1 -weighted MR images were acquired by a gradient-recalled echo sequence with the following acquisition parameters: $\mathrm{TR} / \mathrm{TE}=12 \mathrm{~ms} / 6 \mathrm{~ms}$, scan time $=3.09 \mathrm{~min}$, FOV $=7 \mathrm{~cm} \times 7 \mathrm{~cm}$, matrix $=256 \times 256, \mathrm{ST}=1 \mathrm{~mm}$, spatial resolution $=0.27 \mathrm{~mm} \times 0.27 \mathrm{~mm} \times 1 \mathrm{~mm}$, interslice distance $=1 \mathrm{~mm}$, number of slices $=60$, and $\mathrm{NA}=1$, flip angle $=15^{\circ}$.

For the DTI acquisition, diffusion weighted images were acquired with $\mathrm{TR} / \mathrm{TE}=4,000 \mathrm{~ms} / 86 \mathrm{~ms}, \mathrm{FOV}=6 \mathrm{~cm} \times 6 \mathrm{~cm}$, $\mathrm{ST}=1.8 \mathrm{~mm}$, inter-slice distance $=2 \mathrm{~mm}$, matrix $=64 \times 64$, in-plane voxel size $=234 \mu \mathrm{m} \times 234 \mu \mathrm{m}, \mathrm{NA}=1, b$-value $=0$ and $1,000 \mathrm{~s} / \mathrm{mm}^{2}$ applied in 15 non-colinear directions.

\section{Immunostaining}

Harvested brain samples of rats were post-fixed in 4\% PFA for $24 \mathrm{~h}$, and then were vitrified in 20 and 30\% sucrose solutions for $24 \mathrm{~h}$ and 3 days, respectively. Coronal brain sections from all subjects were obtained using a cryostat (RM2135, Leica, Wetzlar, DE). IF was performed on cryosections (20 $\mu \mathrm{m})$ : approximately 1.70 to $-4.80 \mathrm{~mm}$ to Bregma according to Paxinos and Watson (1998). In details, brain sections were washed three times with PBS $(\mathrm{pH}=7.4)$. Sections were then blocked from non-specific binding with $10 \%$ normal donkey serum in PBS containing $0.3 \%$ Trition-X-100 (Sigma-Aldrich, St. Louis, MO, United States) for $2 \mathrm{~h}$ at room temperature. Primary antibodies used were: (1) rabbit polyclonal anti-NICD (Abcam, Cambridge, MA, United States; 1:100); (2) mouse monoclonal anti-Nestin (BD Biosciences, Franklin Lakes, NJ, United States; 1:1000); (3) goat monoclonal anti-Dcx (Santa Cruz Biotechnology, Santa Cruz, CA, United States; 1:100); (4) rabbit monoclonal anti-NeuN (Biosensis, Thebarton, SA, Australia; 1:250); (5) mouse polyclonal-anti MBP (Abcam, Cambridge, MA, United States; 1:500). Primary antibodies were incubated overnight with sections at $4^{\circ} \mathrm{C}$ and balanced at room temperature for about $30 \mathrm{~min}$. Sections were then rinsed with PBS 3 times for $5 \mathrm{~min}$ and incubated with the following secondary antibodies: Alexa Fluor 488- and 568- conjugated donkey anti-mouse, anti-rabbit, or anti-goat (Life Technologies, Carlsbad, CA, United States; 1:200). Sections were subsequently rinsed with PBS 3 times for $5 \mathrm{~min}$ and counterstained with DAPI, nuclear stain (Sigma-Aldrich, St. Louis, MO, United States; 1:1000). Finally, all the sections were rinsed with PBS 3 times for $5 \mathrm{~min}$, and coverslipped with mounting medium. Sections from the different groups were respectively processed together in the same batches to minimize staining variability.

\section{Image Processing and Quantitative Analysis \\ Magnetic Resonance Imaging}

Regions of interest (ROIs) including the STR and the IC were selected for analysis of T1 SI or DTI parameters. For T1 SI analysis of STR, the ROI was placed on the striatal ischemic boundary with the T1 MR image, which includes the largest area of the lesion. 

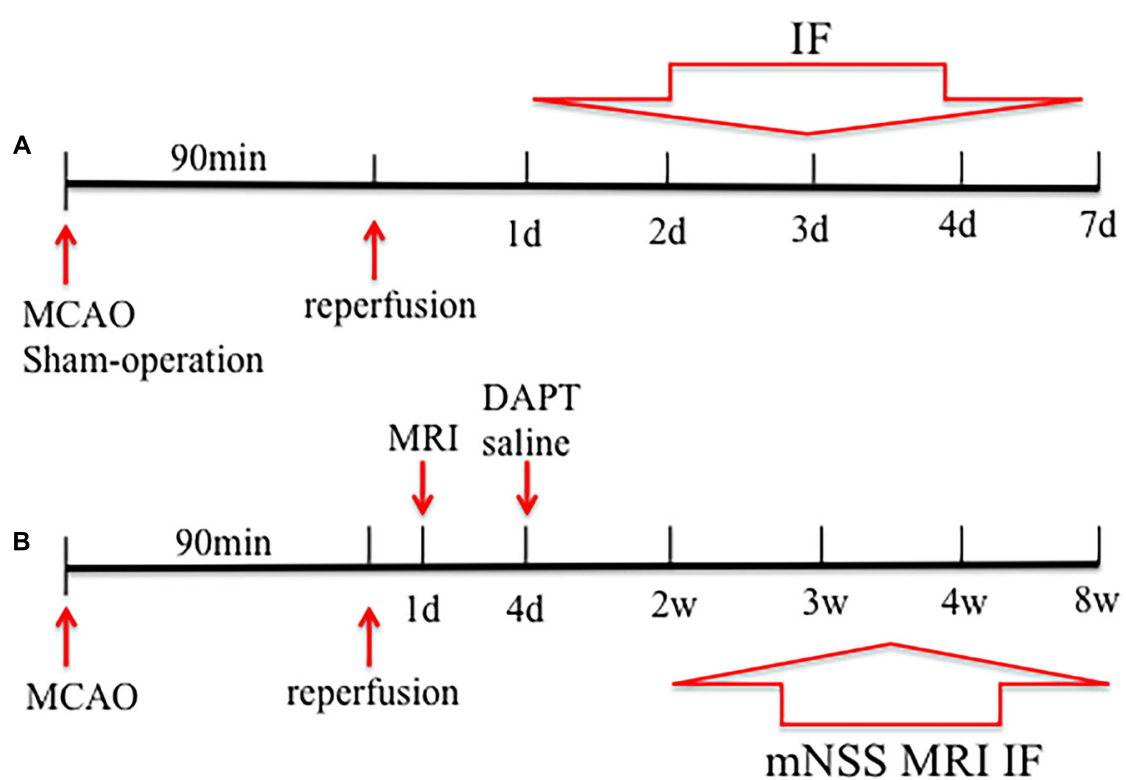

FIGURE 1 | Study design of the experiment. Rats were subjected to middle cerebral artery occlusion (MCAO) in (A,B). (A) Five rats were sacrificed before and 1, 2, 3,4 , and 7 days after stroke, and subjected to immunohistochemical analysis. (B) Rats were treated by DAPT or saline at day4, and eight rats of each subgroup were successively performed with behavioral test, magnetic resonance imaging (MRI) and the subsequent decapitation for immunohistochemical analysis 2 , 3 , 4 , and 8 weeks after stroke.

A

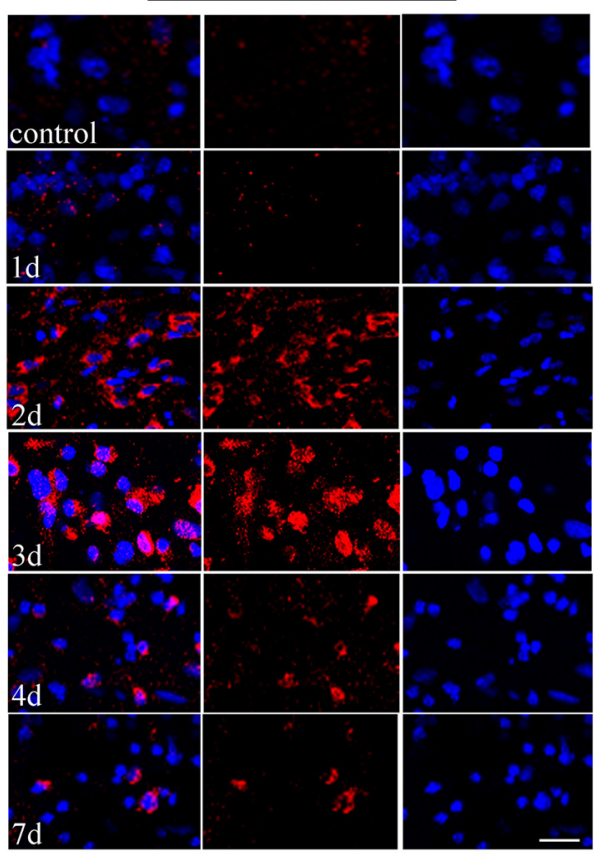

B

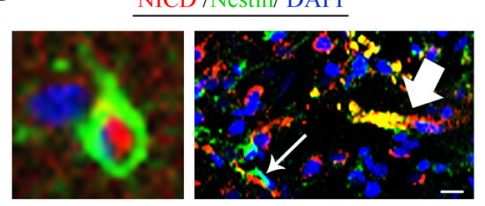

C

NICD /Dcx/ DAPI
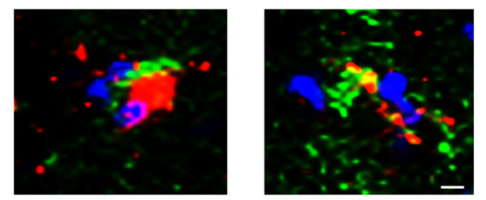

D

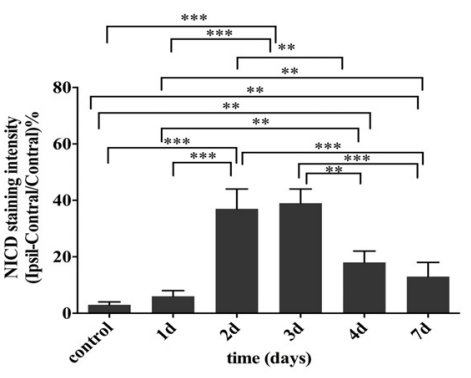

FIGURE 2 | Temporal evolution and spatial location of Noth1 signaling in ischemic striatum (STR). (A) Immunostaining of NICD before and at day 1, day 2, day 3, day 4, and day 7 after MCAO. Scale bar $=20 \mu \mathrm{m}$. (B) Double immunostaining of NICD/Nestin at the ischemic boundary and the lesion of day 3 after MCAO. The thin white arrow and thick white arrow show the double location of the branched neural stem cell (NSC) and vessel-like NSC respectively. Scale bar $=10 \mu \mathrm{m}$. (C) Double immunostaining of NICD/Dcx at the ischemic boundary of day 4 after MCAO. Scale bar $=10 \mu \mathrm{m}$. (D) Quantification of NICD fluorescence intensity in ischemic STR. ${ }^{* * *} P<0.001$ versus day 2 , day 3 and control, day 1 , day $7 .{ }^{* *} P<0.01$ versus day 4 and control, day 1 , day 2 , day $3 .{ }^{* *} P<0.01$ versus day 7 and control, day 1 . 

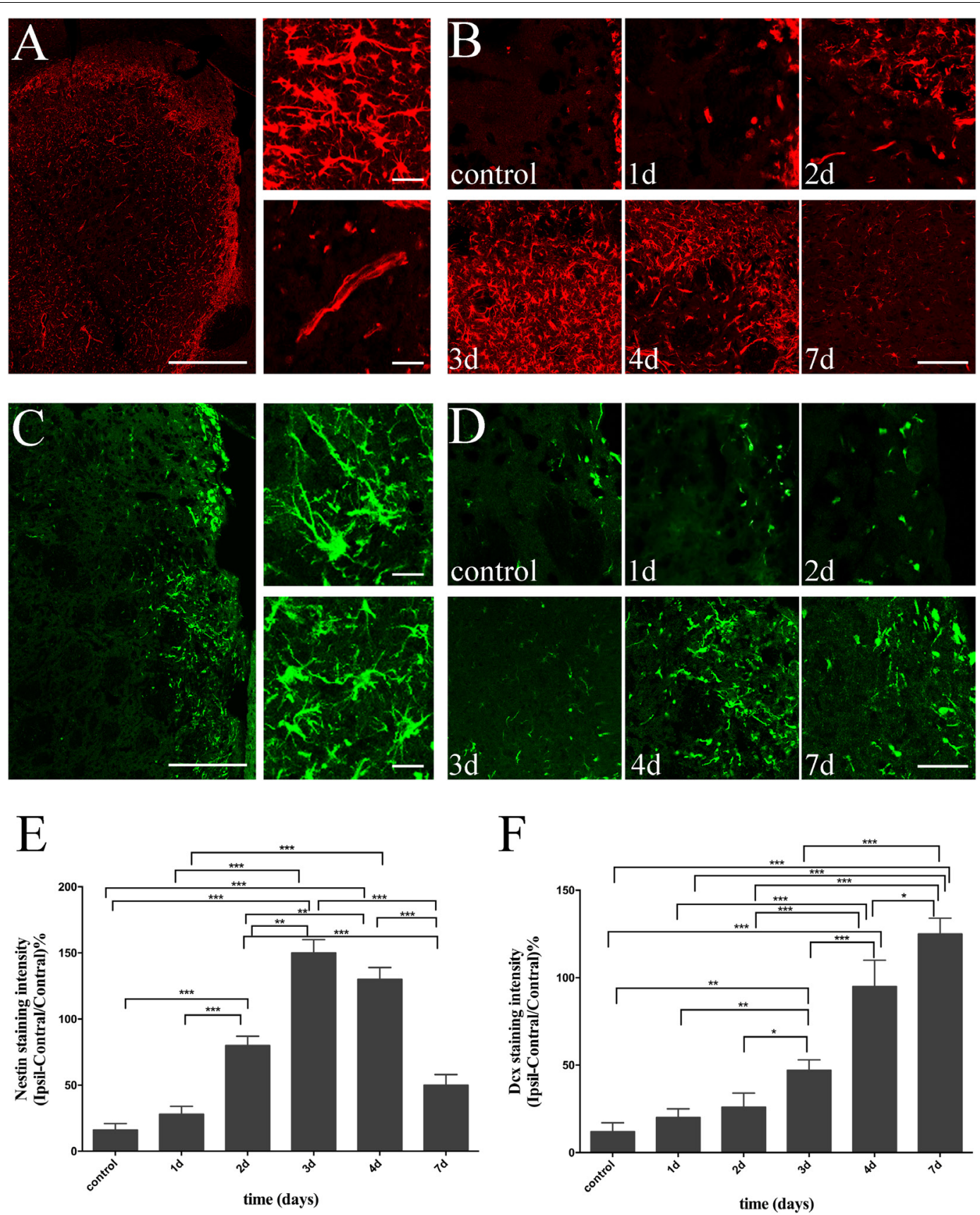

FIGURE 3 | Temporal evolution of NSCs and immature neurons in ischemic STR. (A) The "photomerged" image shows the location of Nestin (+) cells in ischemic STR. The corresponding right panel presents $\times 20$ magnification images of branched and vessel-like NSCs respectively. Scale bar $=100$ and $20 \mu \mathrm{m}$.

(B) Immunostaining of Nestin before and at day 1, day 2, day 3, day 4 and day 7 after MCAO. Scale bar $=50 \mu \mathrm{m}$. (C) The "photomerged" image shows the location of Dcx (+) cells in ischemic STR. The corresponding right panel presents $\times 20$ magnification images of immature neurons be characteristic of elongated processes or riches in processes in different directions respectively. Scale bar $=100$ and $20 \mu \mathrm{m}$. (D) Immunostaining of Dcx before and at day 1, day 2, day 3, day 4, and day 7 after MCAO. Scale bar $=50 \mu \mathrm{m}$. (E) Quantification of Nestin fluorescence intensity in ischemic STR. ${ }^{* * *} P<0.001$ versus day 2 , day 3 , day 4 and control, day 1 , day 7. ${ }^{* *} P<0.001$ versus day 2 and day 3 , day 4 . (F) Quantification of Dcx fluorescence intensity in ischemic STR. ${ }^{* * *} P<0.001$ versus day 4 , day 7 and control, day 1 , day 2, day 3 . ${ }^{*} P<0.01$ versus day 3 and control, day $1 .{ }^{*} P<0.05$ versus day 3 and day $2 .{ }^{*} P<0.05$ versus day 7 and day 4 .

To assess the DTI parameters, axial T2 MR images were used for anatomical references to assess the topography of infarction. DTI measurements including FA, axial diffusivity (AD) and radial diffusivity (RD) were analyzed in the ischemic boundary of STR, as well as the ipsilateral IC. Contralateral ROI regions were drawn on the contralateral hemisphere according to the size and shape of the ipsilateral ROIs. The entire ROI analysis was repeated twice to ensure reproducibility of the results. The percent change of every 
parameter value was computed as: $100 \times\left(\mathrm{X}_{\mathrm{i}}-\mathrm{X}_{\mathrm{c}}\right) / \mathrm{X}_{\mathrm{c}}$, where $\mathrm{X}$ represents an averaged metric.

\section{Immunostaining}

For histological images, sections were scanned with a $\times 20$ primary objective of a Vslide scanning microscope (Nikon, Chiyoda, Tokyo, Japan) with filter sets for DAPI (EX350/50-EM470/40), FITC (EX493/16-EM527/30), and FRITC $(550,620)$. The initial captures were stitched into the Vslide software to create the integrated images of the whole brain. All the images were acquired with a resolution of $1024 \times 1024$ pixels using constant values for laser power, pinhole, digital gain, offset, and scan speed. With Image J (National Institutes of Health, Bethesda, MD, United States), the acquired images were semi-quantitatively measured by optical density of positively stained cells of the selected ROI. In sham-operated and ischemic rats, firstly, the intensity of NICD, Nestin, Dcx of SVZ and STR from 1 day to 7 days was calculated. In vehicle- and DAPT-treated animals, the intensity of Dcx was calculated in the ipsilateral STR using a 0.5 -mm-wide quadratic grid. The intensity of NeuN and MBP in the ischemic boundary of STR and the intensity of MBP in the ipsilateral IC were calculated. Each value was also calculated in the contralateral site equivalent to the ipsilateral ROIs and was also computed as: $100 \times\left(\mathrm{X}_{\mathrm{i}}-\mathrm{X}_{\mathrm{c}}\right) / \mathrm{X}_{\mathrm{c}}$, where $\mathrm{X}$ represents an averaged metric.

\section{Functional Examination}

Vehicle- and DAPT-treated animals were subjected to behavioral tests to assess sensorimotor function 2, 3, 4, and 8 weeks after the induced stroke. The neurological examination had a maximum of 42 points and 0 in normal rats, including postural signs (forelimb flexion, thorax twisting), gait disturbances (circling, climbing, biased movement when pulling the tail or pushing the back), limb placing (forelimb, hindlimb), beam balance, symmetry of muscle tone and strength, sensory function, and spontaneous activity (Reglodi et al., 2003).

\section{Statistical Analysis}

One-way analysis of variance was performed for multiple group comparisons with post hoc LSD tests performed for each of the two groups. Unpaired $t$-tests were performed for two groups comparison at different time points. The correlations between functional test and histological changes were assessed using the Pearson Product correlation analysis. In statistical tests,

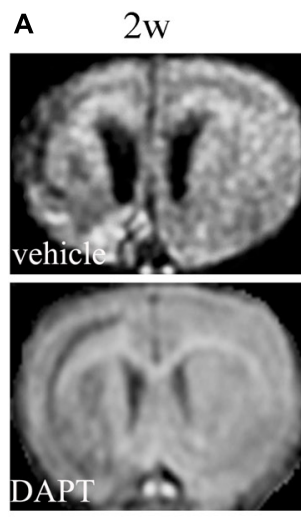

B

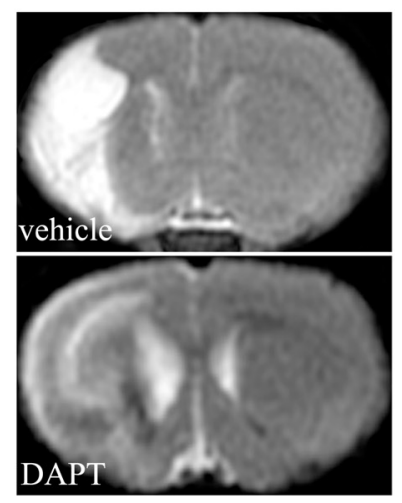

$3 \mathrm{w}$
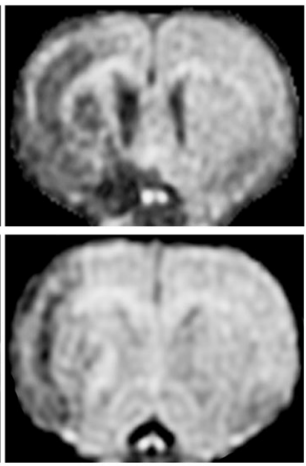

C

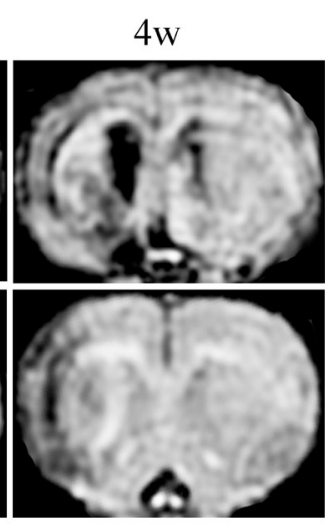

STR

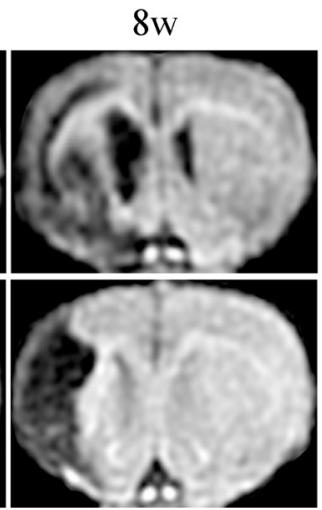

vehicle

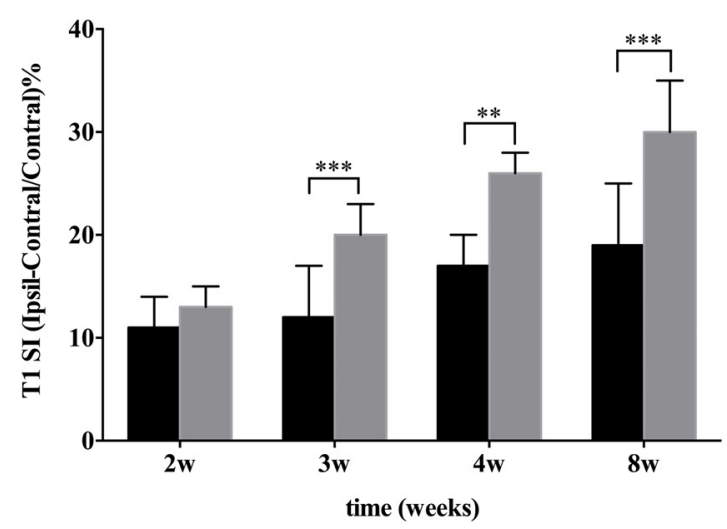

FIGURE 4 | Effects of DAPT on structure changes of STR with T1 MRI after MCAO. (A) The evolution changes in T1 MR images in DAPT-treated rats and vehicle rats from 2 to 8 w. (B) T2 MR images of DAPT-treated rats and vehicle rats at 8 w. (C) Comparison of T1 signal intensity of ischemic boundary between two group rats. ${ }^{* * *} P<0.001,3 \mathrm{w}$; ${ }^{* *} P<0.01,4 \mathrm{w}$; ${ }^{* *} P<0.001,8 \mathrm{w}$ versus DAPT and vehicle. 

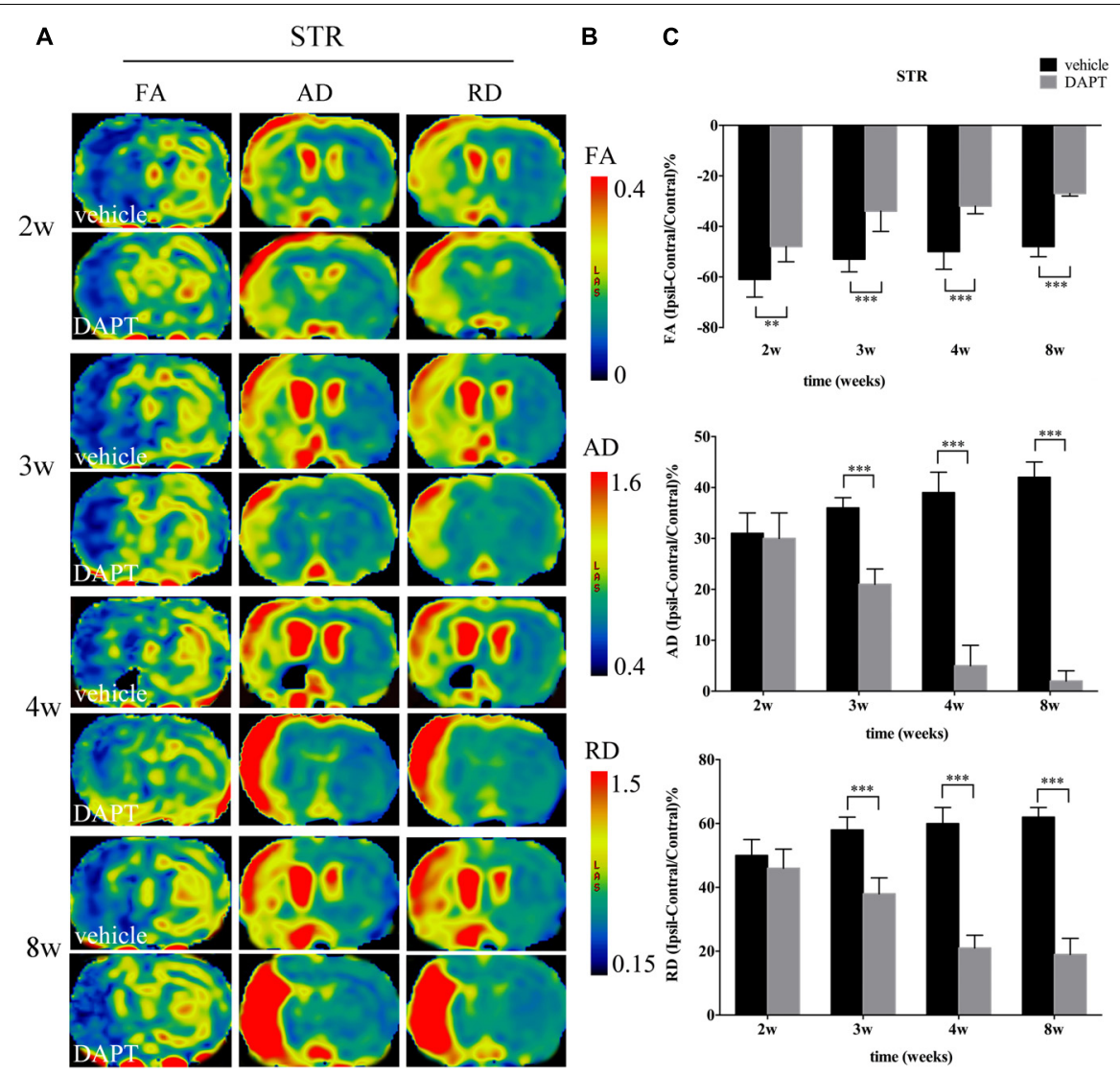

FIGURE 5 | Effects of DAPT on microstructure changes of ischemic boundary of STR with DTI maps after MCAO. (A) The evolution changes in FA, AD and RD of ipsilateral STR in DAPT-treated rats and vehicle rats from 2 to $8 \mathrm{~W}$. (B) The range of FA, AD, and RD. (C) Comparisons of FA, AD, and RD of ischemic boundary of STR between two group rats. FA: ${ }^{* *} P<0.01,2 \mathrm{w} ;{ }^{* * *} P<0.001,3 \mathrm{w}, 4 \mathrm{w}, 8 \mathrm{w}$ versus DAPT and vehicle. AD, RD: ${ }^{* * *} P<0.001,3 \mathrm{w}, 4 \mathrm{w}, 8 \mathrm{w}$ versus DAPT and vehicle.

differences were considered significant when $P<0.05$ and data were presented as the mean $\pm \mathrm{SD}$. The statistical analysis was performed using Prism, version 6.0 (GraphPad Software Inc., La Jolla, CA, United States).

\section{RESULTS}

\section{Notch1 Signal, NSCs, and Neuroblasts Responses After Stroke Activation of Notch1 Signaling}

First, we evaluated the activity of Notch1 signaling at the acute and subacute phase of ischemia by histochemical analysis of the NICD from day 1 to day 7 after the induced stroke (Figure 2A). The intensity of NICD was significantly elevated 2 and 3 days after reperfusion (versus sham-operated group and 1 day, $P<0.001$ ), and reversely decreased 4 days (versus 2 and 3 days, $P<0.01$ ) and 7 days later (versus 2 and 3 days, $P<0.001$ ) later (Figure 2D). Next, to assess whether Notch1 signaling was activated in neural progenitors and neuroblasts after MCAO, we double stained the harvested tissue for markers of Notch intracellular domain (NICD), NSCs (Nestin) and neuroblasts (Dcx). Our results showed insignificant staining for NICD, Nestin and Dcx in sham-operated rats, while in ischemic rats, NICD $(+)$ cells were increased in the ipsilateral STR, where a few of NICD/Nestin $(+)$ cells and NICD/Dcx $(+)$ cells were detected 3 and 4 days after reperfusion. In addition, the NICD was found to be expressed in both the body and the branches of the branched Nestin (+) cells (Figure 2B, thin white arrow) or Dcx $(+)$ cells (Figure 2C), as well as in the vessel-like Nestin $(+)$ cells (Figure 2B, thick white arrow), as indicated in the representative images taken 4 days after reperfusion.

\section{Distribution and Expression of Nestin and Dcx}

At the same time, we investigated the spatial and temporal evolution of NSCs and neuroblasts following ischemia by analyzing the expression of Nestin and Dcx from day 1 to day 7 after MCAO. Nestin, a specific marker of NSCs, was almost non-detectable in the non-ischemic brain, but present in the lesion core (vessel-like structure), as well as in the ischemic boundary (branched structure) in ischemic brain slices (Figure 3A). Moreover, the intensity of Nestin was statistically elevated 2 days (versus Control, 1 day, $P<0.001$ ), peaked at 3 days (versus control, 1 day, $P<0.001$; versus 2 days, $P<0.01$ ), and statistically decreased 7 days (versus 3 and 4 days, $P<0.001$ ) 

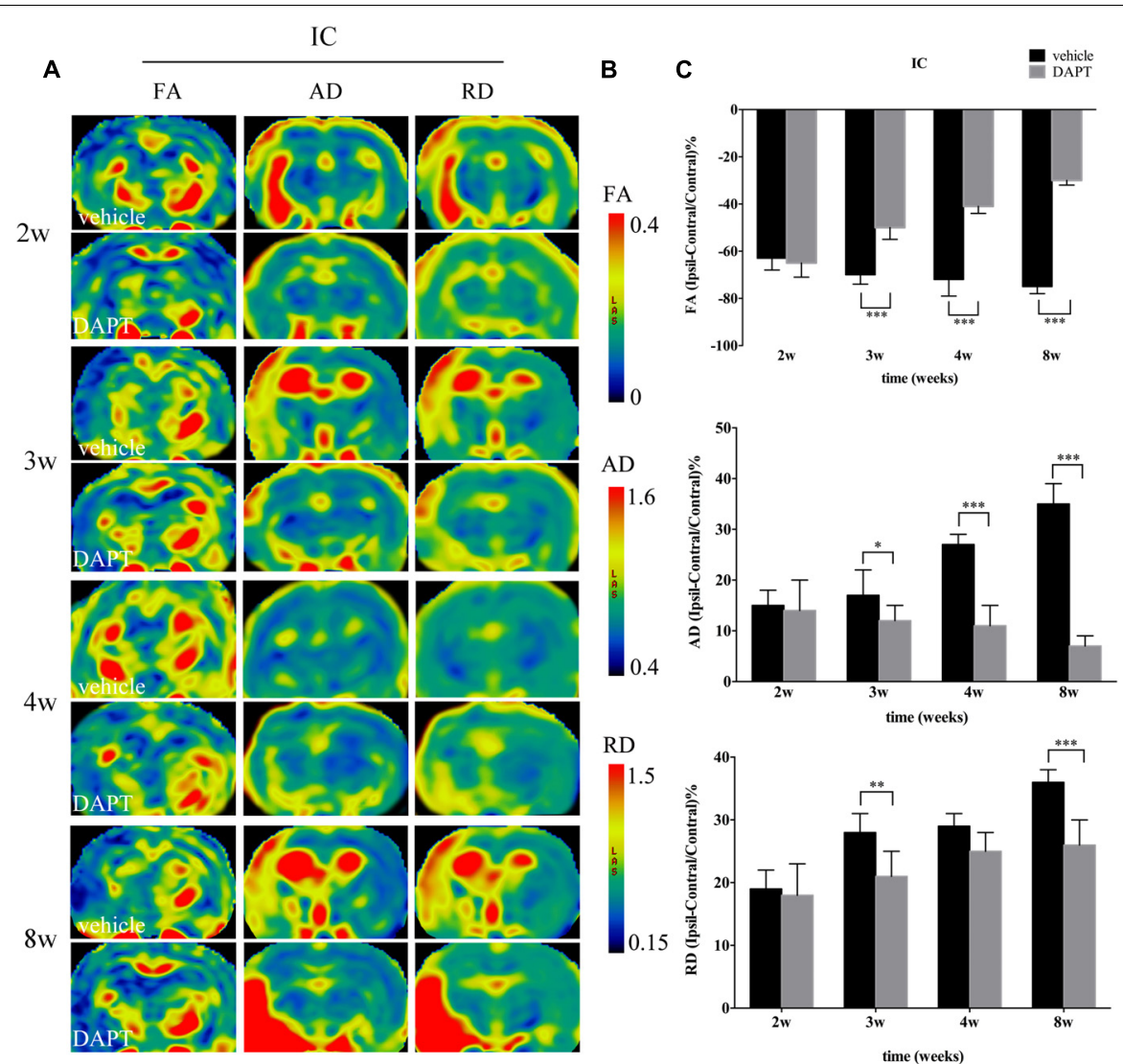

FIGURE 6 | Effects of DAPT on microstructure changes of internal capsule (IC) with DTI maps after MCAO. (A) The evolution changes in FA, AD and RD of ipsilateral IC in DAPT-treated rats and vehicle rats from 2 to $8 \mathrm{~W}$. (B) The range of FA, AD and RD. (C) Comparisons of FA, AD and RD of ipsilateral IC between two group rats. FA: ${ }^{* * *} P<0.001,3 \mathrm{w}, 4 \mathrm{w}, 8 \mathrm{w}$ versus DAPT and vehicle. AD: ${ }^{*} P<0.05,3 \mathrm{w} ;{ }^{* * *} P<0.001,4 \mathrm{w}, 8 \mathrm{w}$ versus DAPT and vehicle. RD: ${ }^{* *} P<0.01,3 \mathrm{w} ;{ }^{* * *} P<0.001,8 \mathrm{w}$ versus DAPT and vehicle.

following reperfusion (Figures 3B,E). Dcx, a specific marker for neuroblasts and differentiating neurons, was detected in the SVZ and in a few striatal cells in sham-operated rats. There were both migrating and non-migrating Dcx $(+)$ cells induced by cerebral ischemia, with the former one being characteristic of elongated and leading processes, while the later one was rich in processes growing in different directions (Figure 3C). Surprisingly, as time followed, we found that migrating Dcx $(+)$ cells were highly distributed in the ischemic STR with their leading processes directed away from the SVZ (Figure 3D). The intensity of Dcx was statistically elevated 3 days later (versus control, 1 day, $P<0.01$; versus 2 days, $P<0.05$ ), significantly increased 4 days later (versus control, 1, 2, and 3 days, $P<0.001$ ), and persisted for 7 days (versus control, 1, 2, and 3 days, $P<0.001$; versus 4 days, $P<0.05$ ) after stroke in the observation time (Figures 3D,F).

\section{Reorganization of White Matter After DAPT Treatment}

\section{T1 SI Changes in Ischemic Boundary}

Measurements of T1 SI were performed on the ischemic boundary of STR, delineated according to the ischemic lesions in T2 MR images, in ischemic animals treated with or without
DAPT (Figures 4A,B). Notably, there was no difference of T1 SI between DAPT-treated versus vehicle-treated animals 2 weeks after the induced stroke, and their differences became significant 3 weeks $(P<0.001)$, 4 weeks $(P<0.01)$, and 8 weeks $(P<0.001)$ following cerebral ischemia (Figure 4C).

\section{DTI Parameter Changes of CST}

Diffusion tension imaging measurements of $\mathrm{FA}, \mathrm{AD}$, and $\mathrm{RD}$ were performed on the ischemic boundary of STR and ipsilateral IC (Figures 5A, 6A), with a consistent range for each value in the two groups (Figures 5B, 6B). In the ischemic boundary zone of STR, FA maps showed a gradual increase in FA at different time points of DAPT- and vehicle-treated rats with differences being significant at 2 weeks $(P<0.01)$. This increase significantly expanded with longer follow-up time points (i.e., from 3 to 8 weeks, $P<0.001$ ) following reperfusion (Figure $5 \mathrm{C}$ ). $\mathrm{AD}$ and $\mathrm{RD}$ measurements exhibited similar temporal profiles, which differed from $\mathrm{FA}$, that $\mathrm{AD}$ and $\mathrm{RD}$ measurements in the ipsilateral ROIs were higher than those in the contralateral side during our observation time in both DAPT- and vehicle-treated animals. However, $\mathrm{AD}$ and $\mathrm{RD}$ represented a gradual increase in vehicle-treated subjects, but a decrease in DAPT-treated group during our observation time period. Importantly, statistically 

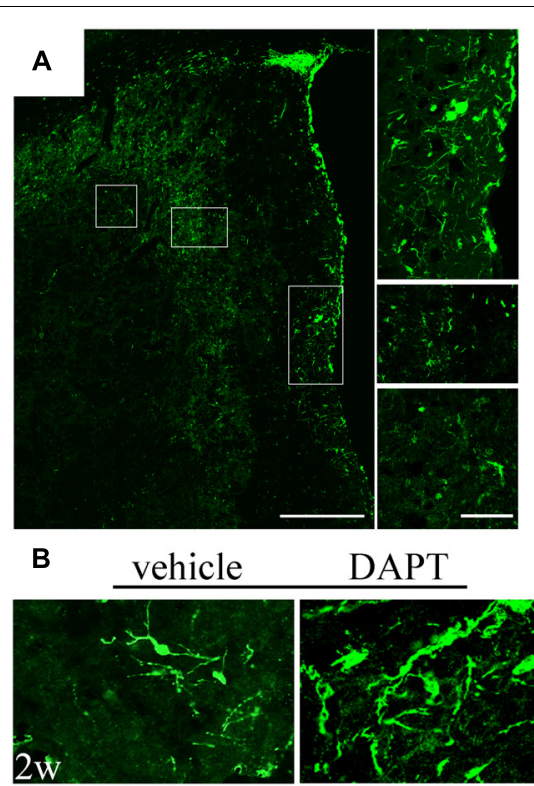

DAPT
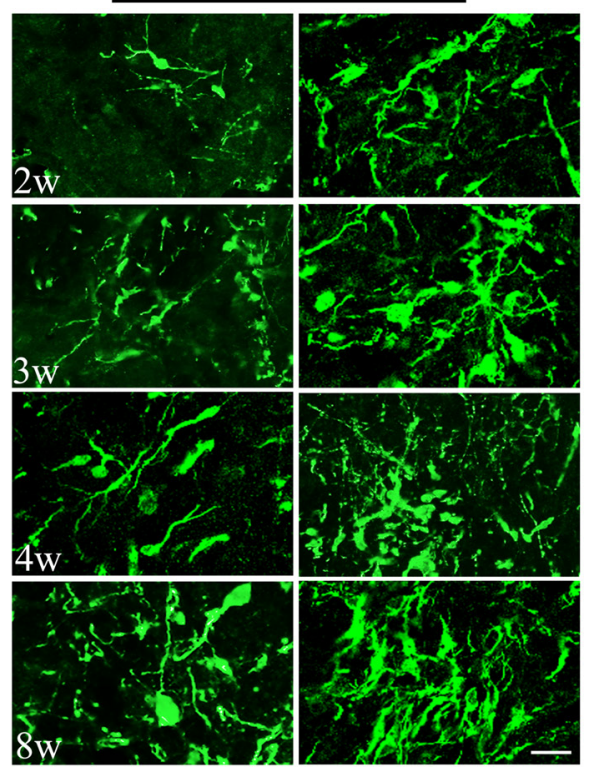

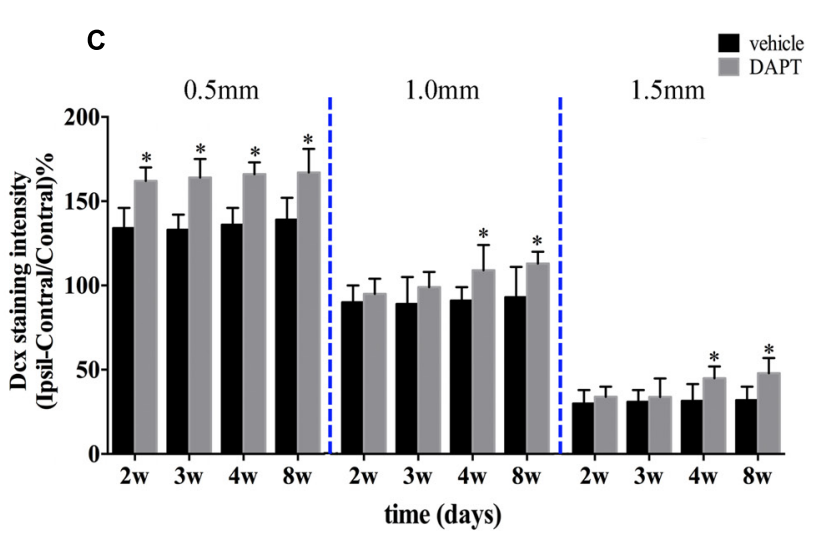

D
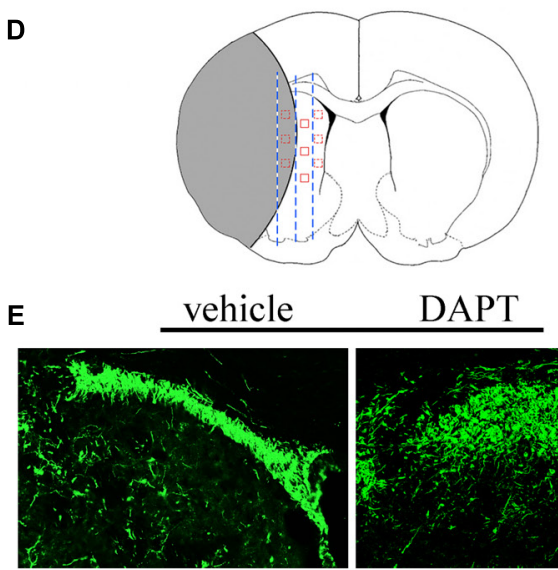

DAPT
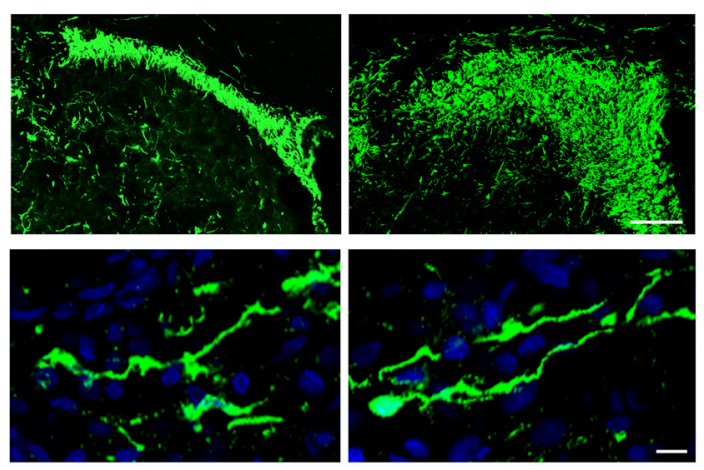

FIGURE 7 | Effects of DAPT on neuroblasts activity after MCAO. (A) Boxes in the "photomerged" image shows the distribution of Dcx (+) cells across the ischemic boundary to the lesion. Scale bar = 100 and $50 \mu \mathrm{m}$. (B) Immunostaining of Dcx inside $0.5 \mathrm{~mm}$ distances from the SVZ in two group rats from 2 to $8 \mathrm{w}$. Scale bar $=20 \mu \mathrm{m}$. (C) Comparisons of Dcx fluorescence intensity in two group rats at different distances from the SVZ 2, 3, 4, and 8 weeks after MCAO. 0.5 mm: ${ }^{*} P<0.05,2,3,4$, 8w versus DAPT and vehicle. $1.0 \mathrm{~mm}, 1.5 \mathrm{~mm}$ : ${ }^{*} P<0.05$, 4w, 8w versus DAPT and vehicle. (D) Anatomical references showing the ROls. (E) Representative images of the location of Dcx (+) cells in the anterior SVZ and shape of Dcx (+) cells in the ischemic boundary of two group rats 8 weeks after MCAO. Scale bar $=50$ and $10 \mu \mathrm{m}$.

significant differences were detected between two the groups (both $\mathrm{AD}$ and $\mathrm{RD}$, from 3 to 8 weeks, $P<0.001$ ) (Figure 5C).

In the ipsilateral IC, FA maps showed opposite temporal profiles between the two subject groups with FA gradually decreasing in the vehicle-treated group while increasing in the DAPT-treated group, showing significant differences from 3 to 8 weeks $(P<0.001)$ (Figure 6 C). Similar to what was observed in the STR, the $\mathrm{AD}$ and $\mathrm{RD}$ measurements in the ipsilateral IC were higher than those of the contralateral hemisphere in both groups during our observation time. AD was gradually increased in the vehicle-treated group but decreased in the DAPT-treated one with these differences becoming significant at 3 weeks $(P<0.05)$, expanding at 4 weeks $(P<0.001)$, and 8 weeks $(P<0.001)$ following reperfusion (Figure 6C). At the same time, RD gradually increased in the two groups, but changed slowly in the DAPT-treated group with statistically significant differences at 3 weeks $(P<0.01)$ and 8 weeks $(P<0.001)$ following reperfusion (Figure 6C).

\section{Stroke-Generated Neurons in Ischemic Boundary}

To confirm whether the improved structural changes in the MR images were attributed to the favored neurogenesis by suppression of Notch1 signaling, we used the marker of Dcx and NeuN (a marker of mature neurons) on the ischemic boundary in both vehicle- and DAPT-treated groups at the early and late periods of the chronic stage of stroke respectively. Dcx $(+)$ cells were found distributed from the SVZ laterally toward the damaged region and presented long lasting processes in all subjects, as seen in the corresponding representative image 

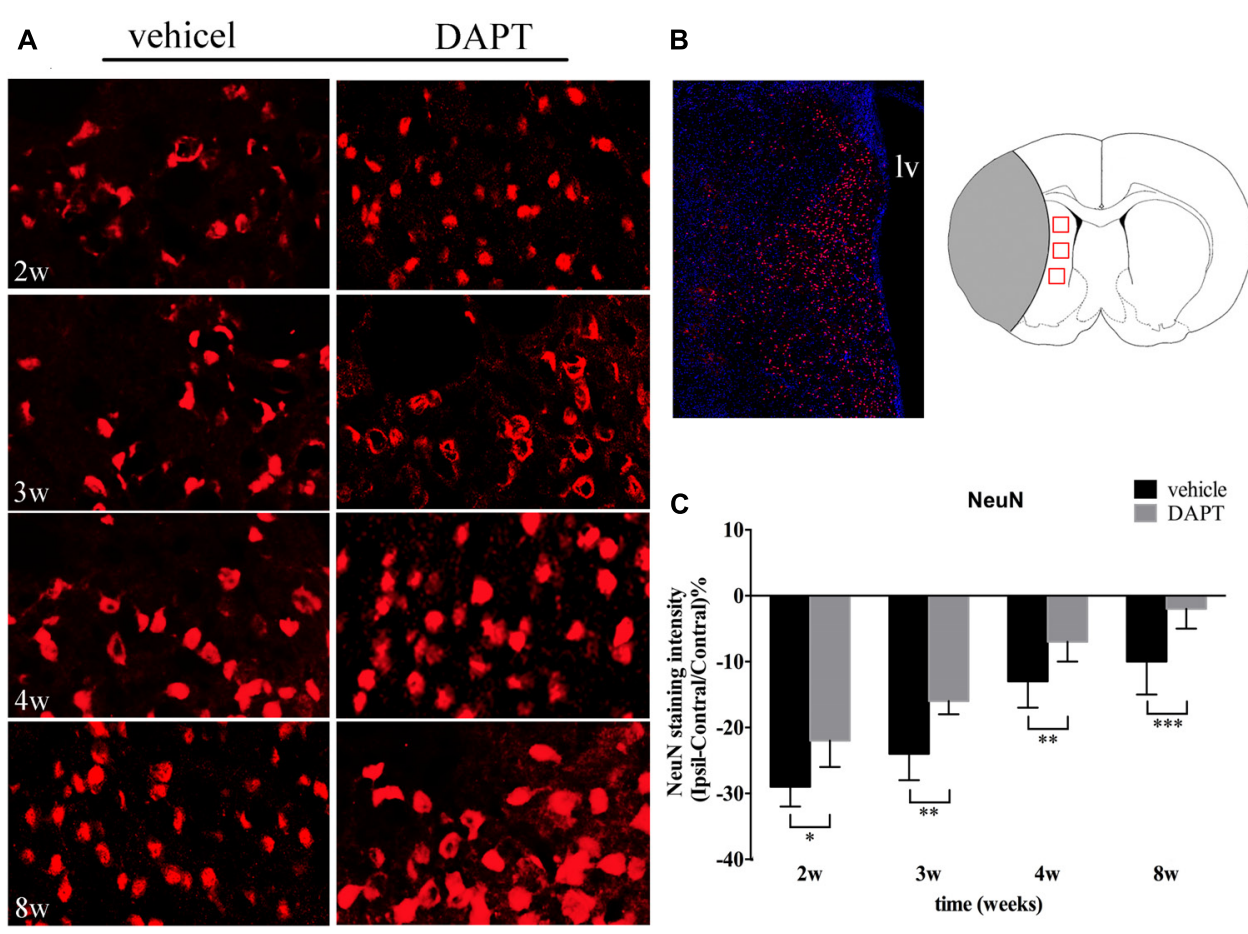

FIGURE 8 | Effects of DAPT on mature neurons activity after MCAO. (A) Immunostaining of NeuN in ischemic boundary of STR in two group rats from 2 to 8 w. Scale bar $=20 \mu \mathrm{m}$. (B) The "photomerged" image shows the location of NeuN (+) cells in the ischemic boundary of STR. The anatomical reference shows the ROls. Scale bar $=100 \mu \mathrm{m}$. (C) Comparisons of NeuN fluorescence intensity in two group rats. ${ }^{*} P<0.05,2 \mathrm{w}$; ${ }^{* *} P<0.01,3 \mathrm{w}, 4 \mathrm{w}$; ${ }^{* * *} P<0.001,8 \mathrm{w}$ versus DAPT and vehicle.

(Figure 7A). Moreover, the SVZ area in the DAPT-treated group presented more Dcx $(+)$ cells, 2-8 weeks after stroke induction (Figure 7B). To detect the effect of DAPT treatment on the proliferation and migration of neuroblasts, we calculated the intensity of Dcx at $0.5,1.0$, and $1.5 \mathrm{~mm}$ from the SVZ (Figures 7C,D). Our results showed that DAPT treatment increased Dcx $(+)$ cells within $0.5 \mathrm{~mm}$ from 2 to 8 weeks $(P<0.05), 1.0 \mathrm{~mm}$ at 4 weeks, 8 weeks $(P<0.05)$, and $1.5 \mathrm{~mm}$ at 4 weeks, 8 weeks $(P<0.05)$. Furthermore, 8 weeks after reperfusion, the DAPT-treated animals harbored more Dcx (+) cells in the anterior SVZ (aSVZ) and presented identical structure of Dcx $(+)$ cells in the ipsilateral STR (Figure 7E).

We next analyzed the intensity of NeuN staining in the ischemic boundary in both groups (Figures 8A,B). Our results showed that the number of neurons in the DAPT-treated animals increased when compared to the vehicle-treated group, 2 weeks $(P<0.05)$, 3 weeks $(P<0.01)$, 4 weeks $(P<0.01)$, and 8 weeks $(P<0.001)$ after stroke (Figure 8C).

\section{Remyelination of CST}

Since newborn neurons play protective roles on the growing axons, we further analyzed the immunoreactivity of the MBP (a protein present in the myelin sheath surrounding axons) in neural pathways of the STR and the IC. In the ipsilateral STR, bundles of axons were mainly located at the ischemic boundary (Figure 9A). The intensity of MBP gradually increased with time in both groups (Figure 9A), which was larger in the
DAPT-treated group, showing statistically significant differences at 4 weeks $(P<0.001)$ and 8 weeks $(P<0.001)$ after reperfusion (Figures 9B,C). In the ipsilateral IC, axons were presented as fibrous structures, which were positive for MBP antibody (Figures 9B,E). And the intensity of the MBP signal increased following DAPT treatment at 2 weeks $(P<0.001), 3$ weeks $(P<0.001)$, 4 weeks $(P<0.05)$, and 8 weeks $(P<0.001)$ after reperfusion (Figures 9D,E).

\section{Correspondence Between MRI and Histological Measures}

The relationships between the reorganized tissue across the ipsilateral CST detected by MRI and its corresponding histological changes at 8 weeks are presented in Figure 10. In the DAPT treated group, the ischemic boundary of the STR presented a higher T1 SI, as well as an early and larger recovery of $\mathrm{FA}, \mathrm{AD}$, and RD measurements, in accordance with an increased number of mature neurons and axon bundles (Figures 10A,C). At the same time, the ipsilateral IC presented an early and larger recovery of $\mathrm{FA}$ and $\mathrm{AD}$, as well as a moderate increase of $\mathrm{RD}$, in accordance with an increase of axonal bundles in the DAPT-treated group (Figures 10B,D).

\section{Functional Status}

At the same time, we explored whether functional recovery after stroke occurs concomitantly with the increased number of mature neurons in the ischemic boundary of the STR and 

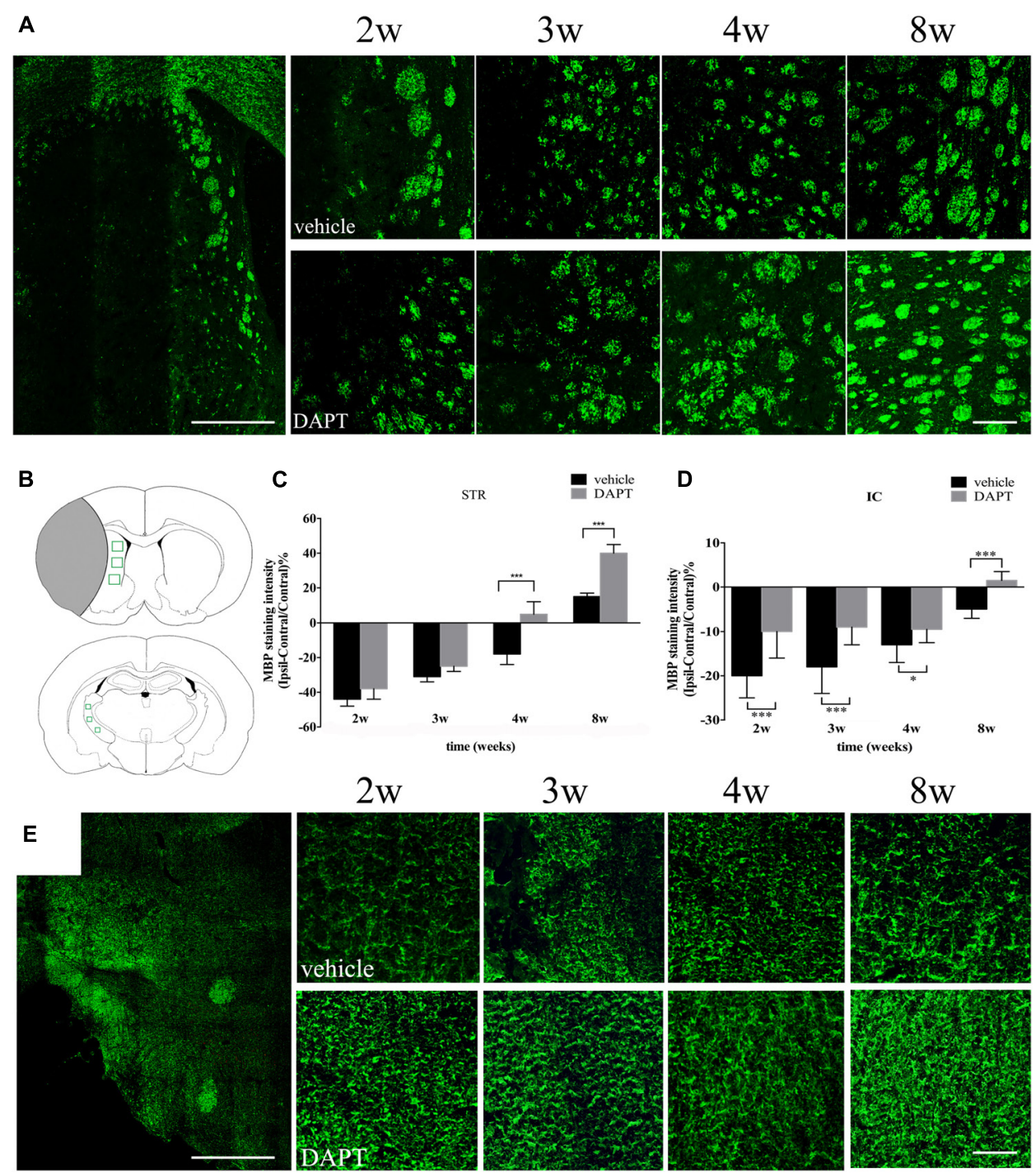

FIGURE 9 | Effects of DAPT on MBP immunoreactivity of STR and IC after MCAO. (A) The "photoemrged" image shows the location of MBP (+) cells in the ipsilateral STR. Immunostaining of MBP in ischemic boundary of STR in two group rats from 2 to $8 \mathrm{w}$. Scale bar $=100$ and $20 \mu \mathrm{m}$. (B) Anatomical references show ROIs in ipsilateral STR and IC respectively. (C) Comparisons of MBP fluorescence intensity of ischemic boundary of STR in two group rats. ${ }^{* * *} P<0.001,4 \mathrm{w}, 8 \mathrm{~W}$ versus DAPT and vehicle. (D) Comparisons of MBP fluorescence intensity of ipsilateral IC in two group rats. ${ }^{* * *} P<0.001,2,3,8 \mathrm{w}$; ${ }^{*} P<0.05,4 \mathrm{w}$ versus DAPT and vehicle. (E) The "photoemrged" image shows the location of MBP (+) cells in the ipsilateral IC. Immunostaining of MBP in ipsilateral IC in two group rats from 2 to $8 \mathrm{~W}$. Scale bar $=100$ and $20 \mu \mathrm{m}$.

the reorganization of myelin sheaths in the ipsilateral CST following DAPT treatment. Functional test examinations showed that animals subjected to DAPT-treatment presented an early and larger motor functional improvement with time (3 weeks, $P<0.01 ; 4$ and 8 weeks, $P<0.001$ ) (Figure 11A). We further analyzed the correlation between functional outcomes at 8 weeks and the corresponding changes in the number of mature neurons as well as the myelin reorganization presented. Our analysis showed a negative correlation between the neurological score and the NeuN staining intensity in the STR in both groups examined, with the differences being more significant in the DAPT-treated group (vehicle, $r=-0.73, P<0.05$; DAPT, $r=-0.88, P<0.01$ ) (Figure 11B). At the same time, negative correlations were found between neurological scores and MBP staining intensity in the STR $(r=-0.78, P<0.05)$ and the IC $(r=-0.78$, $P<0.05)$ in the DAPT-treated group but not in vehicle-control (Figures 11C,D).

\section{DISCUSSION}

In this study, we first investigated the temporal evolution of Notch 1 signaling at the early stage of stroke. Notch1 signaling was found to be maximally activated 3 days after ischemia induction and was reversely attenuated 4 and 7 days after reperfusion. This result is in accordance with the previous study indicating 


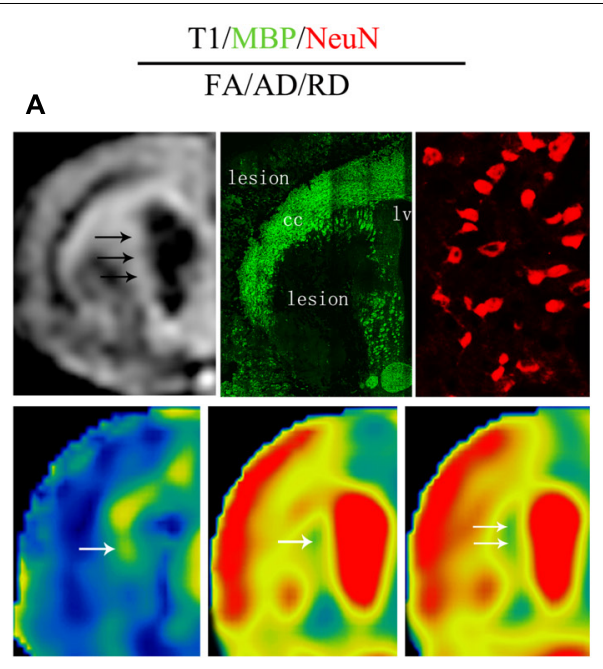

C
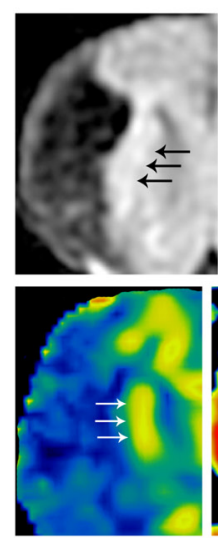
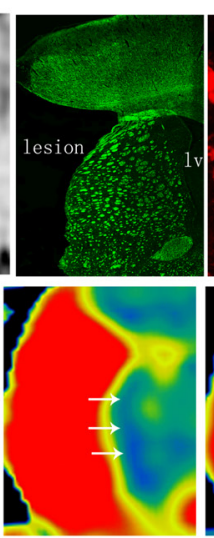
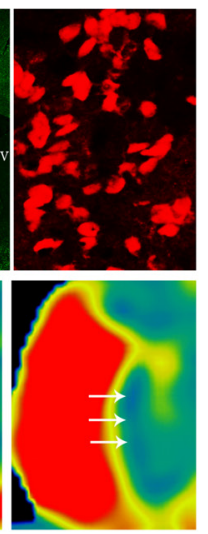

B

T1/FA

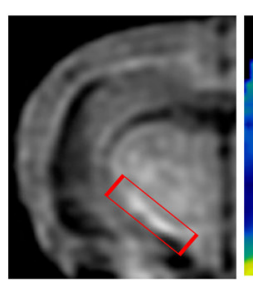

AD/RD/MBP

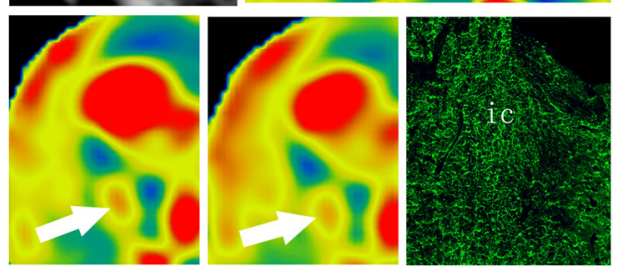

D
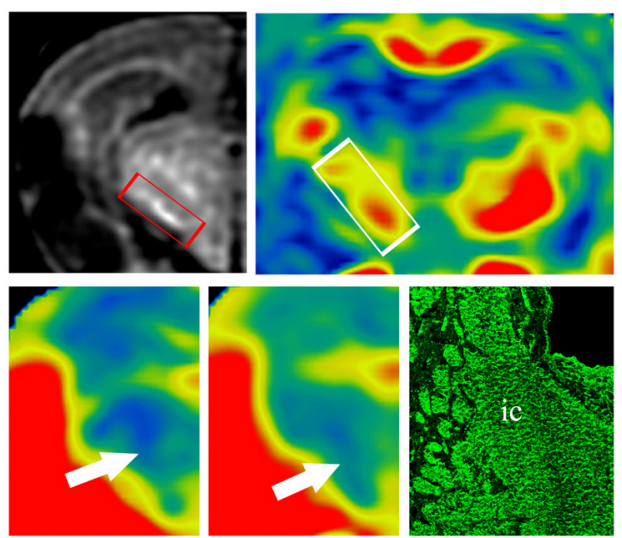

FIGURE 10 | Comprehensive analysis about effects of DAPT on structure-based and cell-based changes in ipsilateral STR and IC 8 weeks after MCAO.

(A) Representative images of T1 MR, MBP, NeuN, FA, AD and RD of ischemic boundary in vehicle rats. (B) Representative images of T1 MR, FA, AD, RD, and MBP of ipsilateral IC in vehicle rats. (C) Representative images of T1 MR, MBP, NeuN, FA, AD, and RD of ischemic boundary in DAPT-treated rats. (D) Representative images of T1 MR, FA, AD, RD and MBP of ipsiateral IC in DAPT-treated rats.

that Notch1 signaling was activated in the acute phase of stroke and then gradually declined in the subacute stage (Oya et al., 2009). Next, we detected the location of NICD in Nestin- and Dcx-positive cells by immunohistochemical analysis, indicating that Notch1 signaling was activated in this cell population and may regulate endogenous neurogenesis.

At the same time, we detected the spatial and temporal evolution of NSCs and neuroblasts at the early stage of stroke. Nestin-positive cells were found to be diffusively located at the lesion and the peri-lesion area of the ipsilateral STR and the ipsilateral cortex. In the ipsilateral STR, there was little expression of Nestin at day 1, which was statistically increased at day 2, peaked at day 3, while reversely declined at day 4 and day 7 following reperfusion. Dcx-positive cells were located at the ipsilateral STR, but not at the ipsilateral cortex, which was consistent with previous studies (Arvidsson et al., 2002). The expression of Dcx statistically significantly increased at day 3 and increased at day 4, day 7 after stroke, which exhibited chains oriented toward the ischemic boundary, formed clusters, and were dispersed in the ipsilateral striatum. Compelling evidences have previously shown that actively proliferating progenitor cells in the SVZ differentiate into migrating neuroblasts that travel to the ischemic boundary (Arvidsson et al., 2002; Thored et al., 2006). The above temporal expression of Nestin and Dcx at the early stage of stroke in our study showed the largest proliferative period of NSCs and the statistically significant increases in neuroblast proliferation at day 3 , indicating the potential transdifferentiation from NSCs to a neuronal lineage. Additionally, the temporal migration of neuroblasts from the SVZ to the ischemic striatum also corresponds to a tendency for neuronal regeneration (Arvidsson et al., 2002; Thored et al., 2006).

Recent studies have demonstrated that early activation of Notch1 signaling activation promotes NSCs proliferation, while late Notch1 signaling activation promotes NSCs differentiation (Oya et al., 2009; Wang L. et al., 2009). The results in our study indicated that the temporal profile of NICD is comparable with the expansion of neural progenitors and the generation 

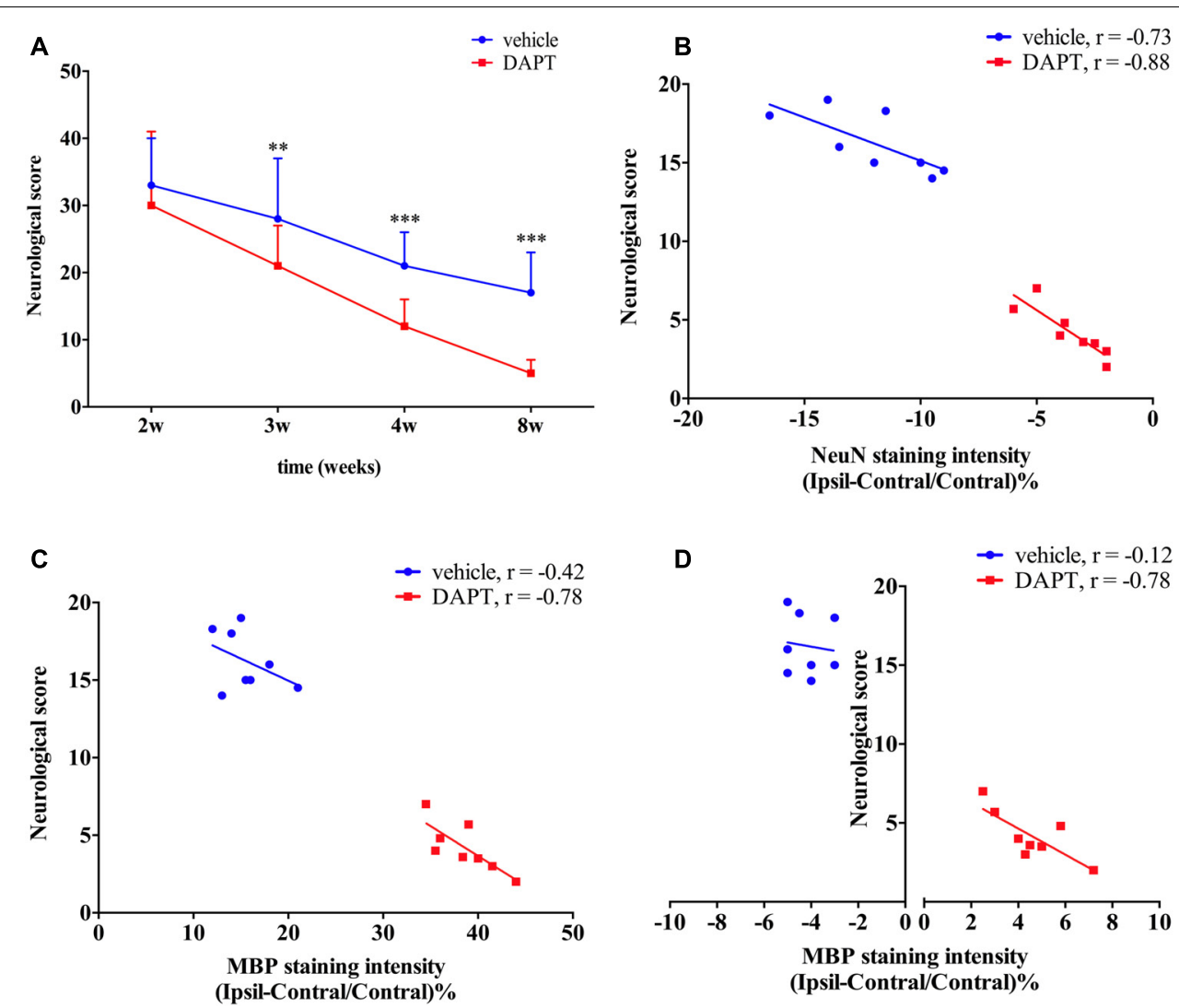

FIGURE 11 | Functional test and correlation analysis. (A) Neurological score. (B) The correlation analyze between neurological score and NeuN staining intensity. (C) The correlation analyze between neurological score and MBP staining intensity of ipsilateral STR. (D) The correlation analyze between neurological score and MBP staining intensity of ipsilateral IC.

of neuroblasts after stroke (Oya et al., 2009; Wang L. et al., 2009). Importantly, blocking Notch1 signaling activation at the appropriate time to expand neural progenitor pools and augment neural differentiation may be a therapeutic intervention with significant benefits to stroke patients. Thus, based on the results of our current study, we hypothesized that a proper therapeutic time point may be 4 days following stroke in this rat model.

In our previous study, we demonstrated that Notch1 signaling could be blocked by DAPT at day 4 after stroke (Hao et al., 2017). Based on this work, we used the same method in this study, to evaluate microstructural changes of rat brains in vivo at chronic stages of stroke by monitoring a set of MRI parameters in the CST both proximal and distal to the ischemic lesion. DTI measurements alone are not sufficient to tackle the complex pathology underlying ischemic processes, additional contrasts, such as T1 and T2 MRI images, should be included in future study designs, together with an integration of the data presented here, could provide complementary information about the status of ischemic tissue following stroke (Jacobs et al., 2001; Wegener et al., 2006). In this study, we firstly analyzed the T1 MR images to distinguish and stage the ischemic boundary changes, and further characterized the microstructure details with both the T1 SI and diffusion MR parameters (FA, $\mathrm{AD}, \mathrm{RD})$. The ischemic boundary in the STR presented a gradual increase of T1 SI in all ischemic rats during the chronic stage after stroke, which accounted for various important factors including decreased edema, astrogliosis, and microglial infiltration, as well as neurogenesis and remyelination. Particularly, the larger increase of T1 caused by the DAPT treatment may account for one of the factors mentioned above (Wegener et al., 2006). To further detect the microstructure changes of white matter bundles, we analyzed the diffusion changes of CST, which may allow the prediction of functional outcomes in chronic stroke patients (Stinear et al., 2007). FA is a common DTI parameter that measures the degree of voxel diffusion (Jones et al., 2013; Ferris et al., 2017). AD quantifies diffusivity along the major orientation of the axon bundles (Song et al., 2002), while RD represents diffusivity perpendicular to white matter bundles (Suttner et al., 2016). When a tissue undergoes ischemia, structural networks represent two signatures including perilesional degenerative changes and remote transneuronal changes, which are both characterized by decreased FA and increased AD, RD measurements (Umarova et al., 2017). These changes are accompanied with histological changes including neural shrinkage as well as a reduction of axonal myelin content and fiber number (Umarova et al., 2017). In our study, FA gradually increased in the ipsilateral STR, while decreased in the ipsilateral IC following ischemia, showing a larger increase in 
the STR and an early increase in the IC after DAPT treatment. $\mathrm{AD}$ was increased in both the ipsilateral STR and the IC after stroke, and gradually decreased to baseline levels following DAPT treatment. Similarly, RD increased in the ipsilateral STR and the IC after ischemia, and showed early decrease in the STR, as well as a moderate increase in the IC after DAPT treatment. The relative recovery of these diffusion parameters mirrored previously reported motor functional abilities of ischemic rats after DAPT treatment (Puig et al., 2010; Reijmer et al., 2013; Feng et al., 2015; Bigourdan et al., 2016), which are now corroborated with our study, which showed that motor function improved after DAPT treatment when compared to vehicle controls in a rat model of stroke.

Using histological examination, we found that neuroblasts were distributed laterally from the SVZ to the ischemic lesion. At different distances from the SVZ, the intensity of Dcx was increased within $0.5 \mathrm{~mm}$ from 2 to 8 weeks, and increased within 1.0 and $1.5 \mathrm{~mm}$ at 4 and 8 weeks, indicating a promotion in neuroblast proliferation during the entirety of our observation period, as well as the induction of cell migration during the late period. DAPT-treated animals presented a large potential for neurogenesis, harboring an increase number of neuroblasts in the aSVZ. More importantly, our two treated groups presented identical structures of neuroblasts, which suggests that DAPT treatments may not alter the function of neuroblasts. Furthermore, the NeuN staining examination showed an increase of mature neurons in the ischemic boundary of the STR in the DAPT-treated group. Significantly, the MBP staining examination showed that the integrity of the CST was recovered early in both the ischemic boundary and the ipsilateral IC.

In this study, though there was no attenuation of necrosis across the lesion, the motor outcome in the DAPT-treated group was significantly elevated when compared to the vehicle-treated group. The most possible contribution should be attributed to the promotion of neurogenesis following DAPT treatment. First, these newborn striatal neurons integrate with preexisting neurons to form functional synapses in the viable brain tissue (Hou et al., 2008; Sun et al., 2012). Secondly, these newly generated neurons could form functional long axons projecting to remote regions to participate in the restorative processes of brain tissue (Sun et al., 2012). As target regions of CST, the STR and the IC play critical roles in the regulation of motor behavior, in which an increase in neural connections could replace damaged ones and ameliorate motor outcomes after stroke. From a novel and comprehensive perspective, with the non-invasive method of MRI, we provided morphological and functional evidence that DAPT treatment could ameliorate the integrity of axon bundles. The post-mortem histological examination confirmed the increase in numbers of mature

\section{REFERENCES}

Arvidsson, A., Collin, T., Kirik, D., Kokaia, Z., and Lindvall, O. (2002). Neuronal replacement from endogenous precursors in the adult brain after stroke. Nat. Med. 8, 963-970. doi: 10.1038/ $\mathrm{nm} 747$ neurons in the ischemic boundary zone and the promotion of remyelination across the CST.

With DAPT treatment, the increase in numbers of mature neurons and the ultimately improved motor outcome may be attributed to the attenuated inflammatory responses (Hao et al., 2017), which had been demonstrated in our previous work, and the promoted neurogenesis, which was suggested in the current study. Adult neurogenesis following stroke is a sequential and multistep process ranging from neural precursor proliferation, migration toward the ischemic site, and differentiation into the proper neural phenotype (Bonfanti and Peretto, 2007; Oya et al., 2009; Christie and Turnley, 2012). In this process, the Notch1 signaling pathway mediates expansion of the neural progenitor pool and neural differentiation, which is comparable to the temporal profile of NSCs (Wang L. et al., 2009). Inappropriate Notch1 activation may induce not only precocious maturation, but also a developmental stagnation of progenitors (Wang X. et al., 2009). Thus, the appropriate timely control of Notch1 signaling activity could produce a large proliferative pool and further promote neuronal differentiation.

\section{CONCLUSION}

With this study, we provided additional evidence that the adult brain can undergo neural replacement from endogenous precursors to repair itself after stroke, which was shown here to be controlled by the activity of Notch1 signaling. Then with the noninvasive MR method, we detected the protective effects of DAPT treatment at the subacute stage of stroke to maximally promote endogenous neurogenesis and axonal reorganization. Our results may enhance the notion of Notch-1 based therapy efficacy in neural repair and promote its application in clinical trials.

\section{AUTHOR CONTRIBUTIONS}

$\mathrm{X}-\mathrm{ZH}$ carried out the animal experiments, performed the MR scanning, and drafted the manuscript. L-KY processed the MR imaging and carried out the histological analysis. J-QT and C-CL processed the MR imaging. X-YF and Z-WY supervised the MR imaging. MJ performed the histological analysis. Y-MY instructed the study protocol and revised the manuscript. All of the authors read and approved the final manuscript.

\section{FUNDING}

This study was supported by the grant of National Natural Science Foundation of China (Nos. 81771788 and 81371521).

Barone, F. C. (2010). Post-stroke pharmacological intervention promoting brain. Neuropharmacology 59, 650-653. doi: 10.1016/j.neuropharm.2010.08.016

Bigourdan, A., Munsch, F., Coupé, P., Guttmann, C. R. G., Sagnier, S., Renou, P., et al. (2016). Early fiber number ratio is a surrogate of corticospinal tract integrity and predicts motor recovery after stroke. Stroke 47, 1053-1059. doi: $10.1161 /$ strokeaha.115.011576 
Bonfanti, L., and Peretto, P. (2007). Radial glial origin of the adult neural stem cells in the subventricular zone. Prog. Neurobiol. 83, 24-36. doi: 10.1016/j.pneurobio. 2006.11.002

Budde, M. D., and Frank, J. A. (2012). Examining brain microstructure using structure tensor analysis of histological sections. Neuroimage 63, 1-10. doi: 10.1016/j.neuroimage.2012.06.042

Chambers, C. B., Peng, Y., Nguyen, H., Gaiano, N., Fishell, G., and Nye, J. S. (2001). Spatiotemporal selectivity of response to notch1 signals in mammalian forebrain precursors. Development 128, 689-702.

Christie, K. J., and Turnley, A. M. (2012). Regulation of endogenous neural stem/progenitor cells for neural repair-factors that promote neurogenesis and gliogenesis in the normal and damaged brain. Front. Cell. Neurosci. 6:70. doi: 10.3389/fncel.2012.00070

Feng, W., Wang, J., Chhatbar, P. Y., Doughty, C., Landsittel, D., Lioutas, V. A., et al. (2015). Corticospinal tract lesion load: an imaging biomarker for stroke motor outcomes. Ann. Neurol. 78, 860-870. doi: 10.1002/ana.24510

Ferris, J. K., Edwards, J. D., Ma, J. A., and Boyd, L. A. (2017). Changes to white matter microstructure in transient ischemic attack: a longitudinal diffusion tensor imaging study. Hum. Brain Mapp. 38, 5795-5803. doi: 10.1002/hbm. 23768

Hao, X. Z., Tian, J. Q., Yin, L. K., Zhang, X. X., Li, C. C., Feng, X. Y., et al. (2017). MRI detects protective effects of DAPT treatment with modulation of microglia/macrophages at subacute and chronic stages following cerebral ischemia. Mol. Med. Rep. 16, 4493-4500. doi: 10.3892/mmr.2017.7200

Hao, X. Z., Yin, L. K., Zhang, X. X., Tian, J. Q., Li, C. C., Feng, X. Y., et al. (2016). Combining systemic and stereotactic MEMRI to detect the correlation between gliosis and neuronal connective pathway at the chronic stage after stroke. J. Neuroinflammation 13:156. doi: 10.1186/s12974-016-0622-7

Hou, S. W., Wang, Y. Q., Xu, M., Shen, D. H., Wang, J. J., Huang, F., et al. (2008). Functional integration of newly generated neurons into striatum after cerebral ischemia in the adult rat brain. Stroke 39, 2837-2844. doi: 10.1161/ STROKEAHA.107.510982

Jacobs, M. A., Zhang, Z. G., Knight, R. A., Soltanian-Zadeh, H., Goussev, A. V., Peck, D. J., et al. (2001). A Model for multiparametric MRI tissue characterization in experimental cerebral ischemia with histological validation in rat. Stroke 32, 943-949.

Jones, D. K., Knösche, T. R., and Turner, R. (2013). White matter integrity, fiber count, and other fallacies- the do's and don'ts of diffusion MRI. NeuroImage 73, 239-254. doi: 10.1016/j.neuroimage.2012.06.081

Li, S., Zyang, X., Wang, Y., Ji, H., Du, Y., and Liu, H. (2012). DAPT protects brain against cerebral ischemia by down-regulating the expression of Notch 1 and nuclear factor kappaB in rats. Neurol. Sci. 33, 1257-1264. doi: 10.1007/s10072012-0948-6

Lindenberg, R., Zhu, L. L., Rüber, T., and Schlaug, G. (2012). Predicting functional motor potential in chronic stroke patients using diffusion tensor imaging. Hum. Brain Mapp. 33, 1040-1051. doi: 10.1002/hbm.21266

Nucifora, P. G., Verma, R., Lee, S. K., and Melhem, E. R. (2007). Diffusiontensor MR imaging and tractography: exploring brain microstructure and connectivity. Radiology 245, 367-384.

Oya, S., Yoshikawa, G., Takai, K., Tanaka, J. I., Higashiyama, S., Saito, N., et al. (2009). Attenuation of notch signaling promotes the differentiation of neural progenitors into neurons in the hippocampal CA1 region after ischemic injury. Neuroscience 158, 683-692. doi: 10.1016/j.neuroscience.2008.10.043

Paxinos, G., and Watson, C. (1998). The Rat Brain in Stereotaxic Coordinates. San Diego, CA: Academic Press.

Puig, J., Pedraza, S., Blasco, G., Daunis-i-Estadella, J., Prados, F., Remollo, S., et al. (2010). Acute damage to the posterior limb of the internal capsule on diffusion tensor tractography as an early imaging predictor of motor outcome after stroke. AJNR Am. J. Neuroradiol. 32, 857-863. doi: 10.3174/ajnr.A2400

Reglodi, D., Tamas, A., and Lengvari, I. (2003). Examination of sensorimotor performance following middle cerebral artery occlusion in rats. Brain Res. Bull. 59, 459-466.

Reijmer, Y. D., Freeze, W. M., Leemans, A., Biessels, G. J., Utrecht Vascular, Cognitive Impairment, et al. (2013). The effect of lacunar infarcts on white matter tract integrity. Stroke 44, 2019-2021. doi: 10.1161/STROKEAHA.113. 001321

Smajlović, D. (2015). Strokes in young adults- epidemiology and prevention. Vasc. Health Risk Manag. 11, 157-164. doi: 10.2147/VHRM.S53203
Song, S.-K., Sun, S.-W., Ramsbottom, M. J., Chang, C., Russell, J., and Cross, A. H. (2002). Dysmyelination revealed through MRI as increased radial (but Unchanged Axial) diffusion of water. NeuroImage 17, 1429-1436. doi: 10.1006/ nimg.2002.1267

Stinear, C. M., Barber, P. A., Smale, P. R., Coxon, J. P., Fleming, M. K., and Byblow, W. D. (2007). Functional potential in chronic stroke patients depends on corticospinal tract integrity. Brain 130(Pt 1), 170-180. doi: 10.1093/brain/ awl333

Sun, F., Mao, X., Xie, L., Ding, M., Shao, B., and Jin, K. (2013). Notch1 signaling modulates neuronal progenitor activity in the subventricular zone in response to aging and focal ischemia. Aging Cell 12, 978-987. doi: 10.1111/acel. 12134

Sun, X., Zhang, Q. W., Xu, M., Guo, J. J., Shen, S. W., Wang, Y. Q., et al. (2012). New striatal neurons form projections to substantia nigra in adult rat brain after stroke. Neurobiol. Dis. 45, 601-609. doi: 10.1016/j.nbd.2011.09.018

Suttner, L. H., Mejia, A., Dewey, B., Sati, P., Reich, D. S., and Shinohara, R. T. (2016). Statistical estimation of white matter microstructure from conventional MRI. Neuroimage Clin. 12, 615-623. doi: 10.1016/j.nicl.2016.09.010

Thomalla, G., Glauche, V., and Weiller, C. (2005). Time course of wallerian degeneration after ischaemic stroke revealed by diffusion tensor imaging. J. Neurol. Neurosurg. Psychiatry 76, 266-268. doi: 10.1136/jnnp.2004.046375

Thored, P., Arvidsson, A., Cacci, E., Ahlenius, H., Kallur, T., Darsalia, V., et al. (2006). Persistent production of neurons from adult brain stem cells during recovery after stroke. Stem Cells 24, 739-747. doi: 10.1634/stemcells.2005-0281

Umarova, R. M., Beume, L., Reisert, M., Kaller, C. P., Kloppel, S., Mader, I., et al. (2017). Distinct white matter alterations following severe stroke: longitudinal DTI study in neglect. Neurology 88, 1546-1555. doi: 10.1212/wnl. 0000000000003843

Wang, L., Chopp, M., Zhang, R. L., Zhang, L., LeTourneau, Y., Feng, Y. F., et al. (2009). The Notch pathway mediates expansion of a progenitor pool and neuronal differentiation in adult neural progenitor cells after stroke. Neuroscience 158, 1356-1363. doi: 10.1016/j.neuroscience.2008. 10.064

Wang, X., Mao, X., Xie, L., Greenberg, D. A., and Jin, K. (2009). Involvement of notch1 signaling in neurogenesis in the subventricular zone of normal and ischemic rat brain in vivo. J. Cereb. Blood Flow Metab. 29, 1644-1654. doi: $10.1038 /$ jcbfm. 2009.83

Wegener, S., Weber, R., Ramos-Cabrer, P., Uhlenkueken, U., Sprenger, C., Wiedermann, D., et al. (2006). Temporal profile of T2-weighted MRI distinguishes between pannecrosis and selective neuronal death after transient focal cerebral ischemia in the rat. J. Cereb. Blood Flow Metab. 26, 38-47. doi: $10.1038 /$ sj.jcbfm.9600166

Wei, Z., Chigurupati, S., Arumugam, T. V., Jo, D. G., Li, H., and Chan, S. L. (2011) Notch activation enhances the microglia-mediated inflammatory response associated with focal cerebral ischemia. Stroke 42, 2589-2594. doi: 10.1161/ strokeaha.111.614834

Zhan, X., Kuczynski, B., and Sharp, F. R. (2011). Post stroke intervention is the window widening. Neuropharmacology 60, 1000-1002. doi: 10.1016/j. neuropharm.2011.02.004

Zhang, R. L., Zhang, Z. G., and Chopp, M. (2008). Ischemic stroke and neurogenesis in the subventricular zone. Neuropharmacology 55, 345-352. doi: 10.1016/j.neuropharm.2008.05.027

Zhao, Y., Chen, X., Ma, L., Zuo, Z., Zhu, Z., Zhu, X., et al. (2012). Electroacupuncture pretreatment induces tolerance against focal cerebral ischemia through activation of canonical Notch pathway. BMC Neurosci. 13:111. doi: 10.1186/1471-2202-13-111

Conflict of Interest Statement: The authors declare that the research was conducted in the absence of any commercial or financial relationships that could be construed as a potential conflict of interest.

Copyright (C) 2018 Hao, Yin, Tian, Li, Feng, Yao, Jiang and Yang. This is an openaccess article distributed under the terms of the Creative Commons Attribution License (CC BY). The use, distribution or reproduction in other forums is permitted, provided the original author(s) and the copyright owner(s) are credited and that the original publication in this journal is cited, in accordance with accepted academic practice. No use, distribution or reproduction is permitted which does not comply with these terms. 